\title{
Global Dynamics of a Discretized Heroin Epidemic Model with Time Delay
}

\author{
Xamxinur Abdurahman, Ling Zhang, and Zhidong Teng \\ College of Mathematics and System Sciences, Xinjiang University, Urumqi, Xinjiang 830046, China \\ Correspondence should be addressed to Xamxinur Abdurahman; xamxinur@sina.com
}

Received 24 June 2014; Accepted 15 September 2014; Published 20 October 2014

Academic Editor: Wanbiao Ma

Copyright ( 2014 Xamxinur Abdurahman et al. This is an open access article distributed under the Creative Commons Attribution License, which permits unrestricted use, distribution, and reproduction in any medium, provided the original work is properly cited.

\begin{abstract}
We derive a discretized heroin epidemic model with delay by applying a nonstandard finite difference scheme. We obtain positivity of the solution and existence of the unique endemic equilibrium. We show that heroin-using free equilibrium is globally asymptotically stable when the basic reproduction number $R_{0}<1$, and the heroin-using is permanent when the basic reproduction number $R_{0}>1$.
\end{abstract}

\section{Introduction}

As we all know, the use of heroin and other drugs in Europe and more specifically in Ireland and the resulting prevalence are well documented [1-3]. It shows that the use of heroin is very popular and causes many preventable deaths. Heroin is so soluble in the fat cells that it crosses the blood-brain barrier within 15-20 seconds, rapidly achieving a high level syndrome in the brain and central nervous system which causes both the "rush" experience by users and the toxicity. Heroin-related deaths are associated with the use of alcohol or other drugs [4]. Treatment of heroin users is a huge burden on the health system of any country.

We often study infectious diseases with mathematical and statistical techniques; see, for example, [5-11]; however, little has been done to apply this method to the heroin epidemics. In 1979, Mackintosh and Stewart [9] considered an exponential model which is simplified from infectious disease model of Kermack and McKendrick to illustrate how the heroin-using spreads in epidemic fashion. They arranged a numerical simulation to show how the dynamics of spread are influenced by parameters in the model. White and Comiskey [5] attempted to extend dynamic disease modeling to the drug-using career and formulated an ordinary differential equation. They divided the whole population into three classes, namely, susceptible, heroin users, and heroin users undergoing treatment. Their model allows a steady state (constant) solution which represents an equilibrium between the number of susceptible, heroin users, and heroin users in treatment. Furthermore, this ODE model was revisited by Mulone and Straughan [12]; the authors proved that this equilibrium solution is stable both linearly and nonlinearly under the realistic condition in which relapse rate of those in treatment returning to untreated drug use is greater than the prevalence rate of susceptible becoming drug users. Recently, the study of the global properties and permanence of continuous heroin epidemic models attracted the researchers and have some very good results; see [13-16]. Specially, Samanta [15] considered a model with time-dependent coefficients and with different removal rates for three different classes, introduced some new threshold values $R_{*}$ and $R^{*}$, and obtained the permanence of heroin-using career.

Motivated by Samanta [15] and Zhang and Teng [8], we alter a nonautonomous heroin epidemic model with time delay to an autonomous heroin epidemic model. For convenience, we replace $U_{1}$ and $U_{2}$ by $U$ and $V$, respectively. Thus, we obtain the following continuous heroin epidemic model with a distributed time delay:

$$
\begin{aligned}
\dot{S}(t)= & \lambda-\beta_{1}(U) S(t) \int_{0}^{h} U(t-s) d \eta(s) \\
& -\mu_{1} S(t)+\xi_{1} U(t)+\xi_{2} V(t),
\end{aligned}
$$




$$
\begin{aligned}
\dot{U}(t)= & \beta_{1}(U) S(t) \int_{0}^{h} U(t-s) d \eta(s) \\
& +\beta_{3} U(t) V(t)-\left(\mu_{2}+P+\xi_{1}\right) U(t), \\
\dot{V}(t)= & P U(t)-\beta_{3} U(t) V(t)-\left(\mu_{3}+\xi_{2}\right) V(t),
\end{aligned}
$$

where $S(t), U(t)$, and $V(t)$ represent the number of susceptible, heroin users not in treatment, and heroin users in treatment, respectively. We assume that the time taken to become heroin user is $s$. The function $\eta(s):[0, h] \rightarrow$ $[0, \infty)$ is nondecreasing and has bounded variation such that $\int_{0}^{h} \eta(s) d s=\eta(h)-\eta(0)=1$.

For understanding more realistic phenomenon of heroin, a little complicated epidemic model is helpful. By applying Micken's nonstandard discretization method [17] to continuous heroin epidemics model with time delay (1), we derive the following discretized heroin epidemic model with a distributed time delay:

$$
\begin{aligned}
S_{n+1}-S_{n}= & \lambda-\beta_{1}\left(U_{n}\right) S_{n+1} \sum_{k=0}^{h} U_{n-k} \eta_{k} \\
& -\mu_{1} S_{n+1}+\xi_{1} U_{n+1}+\xi_{2} V_{n+1}, \\
U_{n+1}-U_{n}= & \beta_{1}\left(U_{n}\right) S_{n+1} \sum_{k=0}^{h} U_{n-k} \eta_{k} \\
& +\beta_{3} U_{n+1} V_{n+1}-\left(\mu_{2}+P+\xi_{1}\right) U_{n+1}, \\
V_{n+1}-V_{n}= & P U_{n+1}-\beta_{3} U_{n+1} V_{n+1}-\left(\mu_{3}+\xi_{2}\right) V_{n+1},
\end{aligned}
$$

where $S_{n}$ is the susceptible class, $U_{n}$ is the class of heroin users not in treatment, and $V_{n}$ is the class of heroin users in treatment at $n$th step. Since the sufficient condition can be obtained, independently of the choice of a time step-size, we let the time step-size be one for the sake of simplicity. The nonnegative constants $\mu_{1}, \mu_{2}$, and $\mu_{3}$ denote the death rate of the susceptible, heroin users not in treatment, and heroin users in treatment class, respectively. Throughout the paper, it is biologically natural to assume that $\mu_{1} \leq$ $\min \left\{\mu_{2}, \mu_{3}\right\}$. The constant $\lambda>0$ denotes the recruitment rate of susceptible population from the general population. Constant $P>0$ is the proportion of heroin users who enter the treatment class. The individuals in treatment who stop using heroin are susceptible at a constant rate $\xi_{2} \geq 0$. Constant $\beta_{3}$ represents the transmission rate from heroin users in treatment to untreated heroin users. $\beta_{1}\left(U_{n}\right)$ is the probability per unit time and the transmission is used with the form $\beta_{1}\left(U_{n}\right) S_{n+1} \sum_{k=0}^{h} U_{n-k} \eta_{k}$, which includes various delays. By a natural biological meaning, we assume that $\beta_{1}(U)$ is a positive function and that there exists a constant $U_{\beta}>0$ such that $\beta_{1}(U)$ is nondecreasing on the interval $\left[0, U_{\beta}\right]$. The integer $h \geq 0$ is the time delay. The sequence $\eta_{k}:-\infty<\eta_{k}<$ $+\infty(k=0,1, \ldots, h)$ is nondecreasing and has bounded.
The initial conditions of the system (2) are given by

$$
\begin{aligned}
& S_{n}=\psi_{n}^{(1)}, \quad U_{n}=\psi_{n}^{(2)}, \quad V_{n}=\psi_{n}^{(3)}, \\
& \text { for } n=-h,-h+1, \ldots, 0,
\end{aligned}
$$

where $\psi_{n}^{(i)} \geq 0(n=-h,-h+1, \ldots, 0, i=1,2,3)$. Again, by biological meaning, we further assume that $\psi_{0}^{(i)}>0$ for all $i=1,2,3$.

The paper is organized as follows. In Section 2, we prove the positivity and boundedness of the solution of system (2). In Section 3, we deal with the global asymptotic stability of the heroin-using free equilibrium. In Section 4, we consider the permanence of the discrete epidemic model applying Wang's technique. In the discretized epidemic model, sufficient condition for global asymptotic stability and permanence are the same as for the original continuous epidemic model. We give some numerical examples and conclusion in Sections 5 and 6.

\section{Basic Properties}

For system (2), the heroin-using free equilibrium is given by

$$
E^{0}=\left(S^{0}, 0,0\right), \quad S^{0}=\frac{\lambda}{\mu_{1}} .
$$

Define a positive constant $A \equiv \sum_{k=0}^{h} \eta_{k}$. The stability of $E^{0}$ is studied by using the next generation method in [7]. The associated matrix $F$ (of the new heroin-using terms) and the M-matrix $V$ (of the remaining transfer terms) are given as follows, respectively:

$$
F=\left(\begin{array}{cc}
\frac{\beta_{1}(0) \lambda A}{\mu_{1}} & 0 \\
0 & 0
\end{array}\right), \quad V=\left(\begin{array}{cc}
\mu_{2}+P+\xi_{1} & 0 \\
-P & \mu_{3}+\xi_{2}
\end{array}\right)
$$

Clearly, $F$ is nonnegative, $V$ is a nonsingular M-matrix, and $V-F$ has $Z$ sign pattern. The associated basic reproduction number, denoted by $R_{0}$, is then given by $R_{0}=\rho\left(F V^{-1}\right)$, where $\rho$ is the spectral radius of $F V^{-1}$. It follows that

$$
R_{0}=\frac{\beta_{1}(0) \lambda A}{\mu_{1}\left(\mu_{2}+P+\xi_{1}\right)} .
$$

Now, we will consider the positivity and boundedness of solution to system (2). For most continuous epidemic models, positivity of the solution is clear, but, for system (2), the positivity of the sequences $S_{n}, U_{n}$, and $V_{n}$ holds in some condition. 
Lemma 1. Let $\left(S_{n}, U_{n}, V_{n}\right)$ be any solution of system (2) with initial condition (3); then $\left(S_{n}, U_{n}, V_{n}\right)$ is positive for any $n \in N$ and $V_{0}<P\left(1+\mu_{3}+\xi_{2}\right) / \beta_{3}$.

Proof. Let $\left(S_{n}, U_{n}, V_{n}\right)$ be any solution of system (2) with initial condition (3). It is evident that system (2) is equivalent to the following iteration system:

$$
\begin{aligned}
& U_{n+1}=\frac{\beta_{1}\left(U_{n}\right) S_{n+1} \sum_{k=0}^{h} U_{n-k} \eta_{k}+U_{n}}{1-\beta_{3} V_{n+1}+\mu_{2}+P+\xi_{1}}, \\
& V_{n+1}=\frac{V_{n}+P U_{n+1}}{1+\beta_{3} U_{n+1}+\mu_{3}+\xi_{2}}, \\
& S_{n+1}=\frac{\lambda+S_{n}+\xi_{1} U_{n+1}+\xi_{2} V_{n+1}}{1+\beta_{1}\left(U_{n}\right) \sum_{k=0}^{h} U_{n-k} \eta_{k}+\mu_{1}} .
\end{aligned}
$$

In the following, we will use the induction to prove the positivity of solution. When $n=0$, we have

$$
\begin{aligned}
& U_{1}=\frac{\beta_{1}\left(U_{0}\right) S_{1} \sum_{k=0}^{h} U_{-k} \eta_{k}+U_{0}}{1-\beta_{3} V_{1}+\mu_{2}+P+\xi_{2}}, \\
& V_{1}=\frac{V_{0}+P U_{1}}{1+\beta_{3} U_{1}+\mu_{3}+\xi_{2}}, \\
& S_{1}=\frac{\lambda+S_{0}+\xi_{1} U_{1}+\xi_{2} V_{1}}{1+\beta_{1}\left(U_{0}\right) \sum_{k=0}^{h} U_{-k} \eta_{k}+\mu_{1}} .
\end{aligned}
$$

From (8)-(10), we see that, as long as $U_{1}$ is obtained, $V_{1}$ and $S_{1}$ will be obtained too.

If $U_{1}>0$, from (9), we directly obtain $V_{1}>0$ and, from (10), we further obtain that $S_{1}>0$. Furthermore, we also have $N_{1}=S_{1}+U_{1}+V_{1}>0$.

Let $x=U_{1}$; then, from (8)-(10), we see that $x$ satisfies the following equation:

$$
\begin{gathered}
\psi(x)=x-\left(\left(1+\beta_{1}\left(U_{0}\right) \sum_{k=0}^{h} U_{-k} \eta_{k}+\mu_{1}\right) U_{0}\right. \\
\left.\quad+\beta_{1} \sum_{k=0}^{h} U_{-k} \eta_{k} W_{1}(x)\right) \\
\times\left(\left(1+\beta_{1}\left(U_{0}\right) \sum_{k=0}^{h} U_{-k} \eta_{k}+\mu_{1}\right) W_{2}(x)\right)^{-1},
\end{gathered}
$$

where

$$
\begin{aligned}
& W_{1}(x)=\lambda+S_{0}+\xi_{1} x+\xi_{2} V_{1}, \\
& W_{2}(x)=1+\mu_{2}+P+\xi_{1}-\beta_{3} V_{1}, \\
& V_{1}=\frac{V_{0}+P x}{1+\beta_{3} x+\mu_{3}+\xi_{2}} .
\end{aligned}
$$

Substituting $V_{1}$ in $W_{2}(x)$ gets

$$
W_{2}(x)=1+\mu_{2}+\xi_{1}+\frac{P\left(1+\mu_{3}+\xi_{2}\right)-\beta_{3} V_{0}}{1+\mu_{3}+\xi_{2}+\beta_{3} x} .
$$

Since $V_{0}<P\left(1+\mu_{3}+\xi_{2}\right) / \beta_{3}$, we have

$$
W_{2}(0)=1+\mu_{2}+P+\xi_{1}-\frac{\beta_{3} V_{0}}{1+\mu_{3}+\xi_{2}}>0,
$$

and because of $W_{1}(0)=\lambda+S_{0}+\xi_{2} V_{0} /\left(1+\mu_{3}+\xi_{2}\right)>0$, thus

$$
\begin{aligned}
\psi(0)= & -\left(\left(1+\beta_{1}\left(U_{0}\right) \sum_{k=0}^{h} U_{-k} \eta_{k}+\mu_{1}\right) U_{0}\right. \\
& \left.+\beta_{1}\left(U_{0}\right) \sum_{k=0}^{h} U_{-k} \eta_{k} W_{1}(0)\right) \\
& \times\left(\left(1+\beta_{1}\left(U_{0}\right) \sum_{k=0}^{h} U_{-k} \eta_{k}+\mu_{1}\right) W_{2}(0)\right)^{-1}<0 .
\end{aligned}
$$

Substituting $W_{1}$ and $W_{2}$ in $\psi(x)$ yields

$$
\psi(x)=\frac{a x^{2}+b x-c}{\left(1+\beta_{1}\left(U_{0}\right) \sum_{k=0}^{h} U_{-k} \eta_{k}+\mu_{1}\right)(m x+n)} .
$$

Here, constants $a, b, c, m$, and $n$ are as follows:

$$
\begin{aligned}
a= & \left(1+\mu_{2}\right)\left(1+\beta_{1}\left(U_{0}\right) \sum_{k=0}^{h} U_{-k} \eta_{k}+\mu_{1}\right)+\xi_{1}\left(1+\mu_{1}\right)>0 \\
b= & \left(1+\mu_{3}+\xi_{2}\right)\left(1+\mu_{1}\right)\left(1+\mu_{2}+P+\xi_{1}\right) \\
& +\left(1+\mu_{1}\right)\left(\beta_{1}\left(U_{0}\right) \sum_{k=0}^{h} U_{-k} \eta_{k}+\beta_{3} S_{0}-\beta_{3} N_{0}\right) \\
& +\beta_{1}\left(U_{0}\right) \sum_{k=0}^{h} U_{-k} \eta_{k} P\left(1+\mu_{3}\right) \\
& -\beta_{3} \beta_{1}\left(U_{0}\right) \sum_{k=0}^{h} U_{-k} \eta_{k}\left(\lambda+N_{0}\right), \\
c= & \beta_{1}\left(U_{0}\right) \sum_{k=0}^{h} U_{-k} \eta_{k}\left[\left(1+\mu_{3}+\xi_{2}\right)\left(\lambda+N_{0}\right)-\left(1+\mu_{3}\right) V_{0}\right] \\
& -U_{0}\left(1+\mu_{1}\right) \xi_{1}, \\
m= & \beta_{3}\left(1+\mu_{2}+\xi_{1}\right)>0, \\
n= & \left(1+\mu_{2}+P+\xi_{1}\right)\left(1+\mu_{3}+\xi_{2}\right)-\beta_{3} V_{0}>0 .
\end{aligned}
$$


We take the limit on both sides of the above equation:

$$
\begin{aligned}
& \lim _{x \rightarrow+\infty} \psi(x) \\
& =\lim _{x \rightarrow+\infty} \frac{a x^{2}+b x-c}{\left(1+\beta_{1}\left(U_{0}\right) \sum_{k=0}^{h} U_{-k} \eta_{k}+\mu_{1}\right)(m x+n)}=+\infty ;
\end{aligned}
$$

this means that $\psi(x)=0$ has at least one positive solution $\bar{x} \in(0,+\infty)$. So, we have $U_{1}=\bar{x}>0$. Therefore, the positivity of $S_{1}>0, U_{1}>0$, and $V_{1}>0$ is finally obtained. When $n=2$, we have

$$
\begin{aligned}
& U_{2}=\frac{\beta_{1} S_{2} \sum_{k=0}^{h} U_{1-k} \eta_{k}+U_{1}}{1-\beta_{3} V_{2}+\mu_{2}+P+\xi_{1}}, \\
& V_{2}=\frac{V_{1}+P U_{2}}{1+\beta_{3} U_{2}+\mu_{3}+\xi_{2}}, \\
& S_{2}=\frac{\lambda+S_{1}+\xi_{1} U_{2}+\xi_{2} V_{2}}{1+\beta_{1} \sum_{k=0}^{h} U_{1-k} \eta_{k}+\mu_{1}},
\end{aligned}
$$

a similar argument as in the above for $U_{1}, V_{1}$, and $S_{1}$; we also can obtain that $U_{2}>0, V_{2}>0$, and $S_{2}>0$. Lastly, by using the induction, we can finally obtain that $S_{n}>0, U_{n}>0$, and $V_{n}>0$, for all $n>0$.

Now, we define the total population as $N_{n}=S_{n}+U_{n}+V_{n}$. Then, from system (2), we know that

$$
N_{n+1}-N_{n}=\lambda-\mu_{1} S_{n+1}-\mu_{2} U_{n+1}-\mu_{3} V_{n+1} \text {. }
$$

Notice the assumption that $\mu_{1} \leq \min \left(\mu_{2}, \mu_{3}\right)$; we obtain

$$
\begin{aligned}
N_{n} \leq & \frac{\lambda+N_{n-1}}{1+\mu_{1}} \\
\leq & \frac{\lambda}{1+\mu_{1}} \times\left\{1+\frac{1}{1+\mu_{1}}+\cdots+\left(\frac{1}{1+\mu_{1}}\right)^{n-1}\right\} \\
& +\left(\frac{1}{1+\mu_{1}}\right)^{n} N_{0} \\
= & \frac{\lambda}{\mu_{1}}\left\{1-\left(\frac{1}{1+\mu_{1}}\right)^{n}\right\}+\left(\frac{1}{1+\mu_{1}}\right)^{n} N_{0} \\
\leq & \max \left\{\frac{\lambda}{\mu_{1}}, N_{0}\right\} .
\end{aligned}
$$

If $\lambda / \mu_{1} \geq N_{0}$, it is easy to see that $N_{n} \leq \lambda / \mu_{1}=S^{0}$, for all large $n$. If $\lambda / \mu_{1}<N_{0}$, from right hand side of system (2), we obtain

$$
N_{1} \leq \frac{\lambda+N_{0}}{1+\mu_{1}}<N_{0} .
$$

Hence, we have $N_{1}<N_{0}$ and there exists $i \in N$ such that $N_{i} \leq \lambda / \mu_{1}=S^{0}$. Then, we may use this $N_{i}$ as a starting value instead of $N_{0}$. This argument leads to the following result.
Lemma 2. For any solution $\left(S_{n}, U_{n}, V_{n}\right)$ of system (2), the total population $N_{n}=S_{n}+U_{n}+V_{n}$ satisfies

$$
\limsup _{n \rightarrow+\infty} N_{n} \leq S^{0}=\frac{\lambda}{\mu_{1}}
$$

thus $\left(S_{n}, U_{n}, V_{n}\right)$ is ultimately bounded.

Let $\Omega=\left\{\left(S_{n}, U_{n}, V_{n}\right): S_{n}, U_{n}, V_{n} \geq 0, S_{n}+U_{n}+V_{n} \leq\right.$ $\left.\lambda / \mu_{1}\right\}$; then $\Omega$ is the positive invariant set to the solution of system (2).

In the following, we will examine the existence of endemic equilibrium for a special case of system (2).

Lemma 3. Assume that $\beta_{1}(U)=\beta_{1}>0$ is a constant. If $R_{0}>1$, system (2) admits a heroin-using equilibrium $E^{*}=$ $\left(S^{*}, U^{*}, V^{*}\right)$ when $V<P / \beta_{3}$, where $E^{*}$ satisfies following equality:

$$
\begin{gathered}
\lambda-A \beta_{1} S^{*} U^{*}-\mu_{1} S^{*}+\xi_{1} U^{*}+\xi_{2} V^{*}=0, \\
A \beta_{1} S^{*} U^{*}+\beta_{3} U^{*} V^{*}-\left(\mu_{2}+P+\xi_{1}\right) U^{*}=0, \\
P U^{*}-\beta_{3} U^{*} V^{*}-\left(\mu_{3}+\xi_{2}\right) V^{*}=0 .
\end{gathered}
$$

Proof. Consider the following equation:

$$
\begin{gathered}
\lambda-A \beta_{1} S U-\mu_{1} S+\xi_{1} U+\xi_{2} V=0, \\
A \beta_{1} S U+\beta_{3} U V-\left(\mu_{2}+P+\xi_{1}\right) U=0, \\
P U-\beta_{3} U V-\left(\mu_{3}+\xi_{2}\right) V=0 .
\end{gathered}
$$

From the first equation and the second equation of the system (25), we have

$$
\lambda-\mu_{1} S+\xi_{2} V+\beta_{3} U V-\left(\mu_{2}+P\right) U=0 .
$$

From the second equation and the third equation of the system (25), we obtain

$$
A \beta_{1} S U-\left(\mu_{2}+\xi_{1}\right) U-\left(\mu_{3}+\xi_{2}\right) V=0
$$

thus,

$$
U=\frac{\left(\mu_{3}+\xi_{2}\right) V}{A \beta_{1} S-\mu_{2}+\xi_{1}} .
$$

Since $U \neq 0$, from the second equation of the system, we have

$$
S=\frac{\mu_{2}+P+\xi_{1}-\beta_{3} V}{A \beta_{1}} .
$$


Substituting $S$ in (28), we obtain

$$
U=\frac{\left(\mu_{3}+\xi_{2}\right) V}{P-\beta_{3} V}
$$

Substituting $U$ and $S$ in (26) yields a quadratic equation of $V$ as follows:

$$
F(V)=a V^{2}+b V+c=0
$$

where the coefficients are given by

$$
\begin{aligned}
& a=\mu_{1} \beta_{3}^{2}-A \beta_{1} \beta_{3} \mu_{3}, \quad c=\mu_{1} P\left(\mu_{2}+P+\xi_{1}\right)-\lambda A \beta_{1} P, \\
& b=\lambda A \beta_{1} \beta_{3}-\mu_{1} \beta_{3}\left(\mu_{2}+\xi_{1}\right)+A \beta_{1} \mu_{3}\left(\mu_{2}+P\right)+A \beta_{1} \mu_{2} \xi_{2} .
\end{aligned}
$$

Since $R_{0}=\lambda A \beta_{1} / \mu_{1}\left(\mu_{2}+P+\xi_{1}\right)>1$, then it is easy to see that $c<0$ and $b>0$. According to Descartes' rule of signs, if $a \geq 0$, then $F(V)=0$ has a positive solution; if $a<0$, then $F(V)=0$ has two positive solutions. From the expression of $S$ and $U$, we note that $V<P / \beta_{3}$. Since

$$
F(0)=c<0, \quad F\left(\frac{P}{\beta_{3}}\right)=\frac{A \beta_{1} P \mu_{2}\left(\mu_{3}+\xi_{2}\right)}{\beta_{3}}>0 .
$$

This means that $F(V)=0$ has a unique positive solution $V^{*} \in\left(0, P / \beta_{3}\right)$. Therefore, there exists a unique positive solution $\left(S^{*}, U^{*}, V^{*}\right)$ of system (2).

For the local stability of the equilibria, we refer to Theorem 2 in [7] and have the following results.

Theorem 4. Assume that $\beta_{1}(U)=\beta_{1}, \beta_{1}$ is a positive constant. The heroin-using free equilibrium $E^{0}=\left(S^{0}, 0,0\right)$ of system (2) is locally asymptotically stable if $R_{0}<1$ and unstable if $R_{0}>1$.

\section{Global Asymptotic Stability of the Heroin- Using Free Equilibrium}

In this section, we still assume that $\beta_{1}(U)=\beta_{1}>0$, and obtain a sufficient condition for global asymptotic stability of the heroin-using free equilibrium $E^{0}$ of system (2).

Using a Lyapunov function similar to that in [11], we can easily prove the global asymptotic stability of the heroinusing free equilibrium $E^{0}$.

Theorem 5. If $R_{0}<1$, the drug-using free equilibrium $E^{0}$ of system (2) is globally asymptotically stable.

Proof. Let us take the following Lyapunov function:

$$
H_{n}=U_{n}+c_{1} V_{n}+c_{2} \sum_{k=0}^{h}\left(\sum_{l=n-k}^{n} U_{l}\right) \eta_{k}+\frac{c_{3}}{2}\left(S_{n+1}-S^{0}\right)^{2}
$$

where $c_{i}>0(i=1,2,3)$ are the constants to be defined later and $S^{0}=\lambda / \mu_{1}$. Using system (2), the difference of $H_{n}$ satisfies

$$
\begin{aligned}
\Delta H= & H_{n+1}-H_{n} \\
= & U_{n+1}-U_{n}+c_{1}\left(V_{n+1}-V_{n}\right) \\
& +c_{2} \sum_{k=0}^{h}\left(U_{n+1} \eta_{k}-U_{n-k} \eta_{k}\right) \\
& +\frac{c_{3}}{2}\left\{\left(S_{n+1}-S^{0}\right)^{2}-\left(S_{n}-S^{0}\right)^{2}\right\} .
\end{aligned}
$$

From $S_{n} \leq N_{n} \leq S^{0}$, for all $n \geq 0$, we have

$$
\begin{aligned}
\Delta H \leq & \sum_{k=0}^{h} U_{n-k} \eta_{k}\left\{\beta_{1} S_{n+1}-c_{2}-c_{3} \beta_{1} S_{n+1}\left(S_{n+1}-S^{0}\right)\right\} \\
& +\left(1-c_{1}\right) \beta_{3} U_{n+1} V_{n+1} \\
& +\left[c_{2} A-\mu_{2}-P-\xi_{1}+c_{1} P+c_{3} \xi_{1}\left(S_{n+1}-S^{0}\right)\right] U_{n+1} \\
& -\left[c_{1}\left(\mu_{3}+\xi_{2}\right)-c_{3} \xi_{2}\left(S_{n+1}-S^{0}\right)\right] V_{n+1} \\
& -c_{3} \mu_{1}\left(S_{n+1}-S^{0}\right)^{2} \\
\leq & \sum_{k=0}^{n} U_{n-k} \eta_{k}\left\{\beta_{1} S_{n+1}-c_{2}-c_{3} \beta_{1} S_{n+1}\left(S_{n+1}-S^{0}\right)\right\} \\
& +\left(1-c_{1}\right) \beta_{3} U_{n+1} V_{n+1} \\
& +\left[c_{2} A-\mu_{2}-P-\xi_{1}+c_{1} P\right] U_{n+1}-c_{1}\left(\mu_{3}+\xi_{2}\right) V_{n+1} \\
& -c_{3} \mu_{1}\left(S_{n+1}-S^{0}\right)^{2} .
\end{aligned}
$$

Let us choose $c_{i}>0(i=1,2,3)$ such that these constants satisfy the following inequalities:

$$
\begin{gathered}
\beta_{1} S_{n+1}-c_{2}-c_{3} \beta_{1} S_{n+1}\left(S_{n+1}-S^{0}\right)<0 \\
1-c_{1}<0 \\
c_{1} P+c_{2} A<\mu_{2}+P+\xi_{1} .
\end{gathered}
$$

From (37), we have $c_{3} \beta_{1} S_{n+1}^{2}+\left(c_{3} \beta_{1} S^{0}-\beta_{1}\right) S_{n+1}+c_{2}>0$; since $S_{n+1}>0$, then the following inequality is true:

$$
\beta_{1}\left(1+c_{3} S^{0}\right)^{2}<4 c_{2} c_{3}
$$

that is,

$$
\beta_{1}\left(S^{0}\right)^{2} c_{3}^{2}+\left(2 \beta_{1} S^{0}-4 c_{2}\right) c_{3}+\beta_{1}<0
$$

Since $R_{0}<1$, which implies that $\beta_{1} A S^{0}<\mu_{2}+P+\xi_{1}$, we can choose $c_{2}=\beta_{1} S^{0}+\epsilon$; here, $\epsilon\left(0<\epsilon<\left(\mu_{2}+\xi_{1}-A \beta_{1} S^{0}\right) / A\right)$ is a sufficiently small positive number such that $\beta_{1} A S^{0}+A \epsilon<\mu_{2}+$ $\left(1-c_{1}\right) P+\xi_{1}$. Since $\beta_{1} S^{0}-2 c_{2}<0$ and $\left(\beta_{1} S^{0}-2 c_{2}\right)^{2}>\left(\beta_{1} S^{0}\right)^{2}$, we can choose $c_{3}>0$ to satisfy (41). We may further choose $c_{1}>1$ to satisfy (38). Therefore, $\Delta H$ is negative definite and is equal to zero if and only if $S_{n+1}=S^{0}, U_{n+1}=0$, and $V_{n+1}=0$. The proof is complete. 


\section{Permanence of System (2)}

The system (2) is said to be permanent if there are positive constants $m$ and $M$ such that

$$
m \leq \liminf _{n \rightarrow \infty} S_{n} \leq \limsup _{n \rightarrow \infty} S_{n} \leq M
$$

hold for any sequence $S_{n}$ of the system (2), and the same inequalities hold for $U_{n}$ and $V_{n}$. For each class $S_{n}, U_{n}$, and $V_{n}$, $m$ and $M$ are independent of initial conditions.

Following the method used by Wang in [6], we will prove the permanence of system (2) for the general case; that is, assume that $\beta_{1}(U)$ is related to $U$.

Theorem 6. If $R_{0}>1$, then system (2) is permanent for any initial condition (3).

Proof. Firstly, from system (2) and Lemmas 1 and 2, for any $\epsilon_{0}>0$, there exists sufficiently large $n_{0}>0$ such that $U_{n} \leq$ $\lambda / \mu_{1}+\epsilon_{0}$ as $n \geq n_{0}+h$. Then, we have

$$
\begin{aligned}
S_{n+1} & =\frac{\lambda+S_{n}+\xi_{1} U_{n+1}+\xi_{2} V_{n+1}}{1+\beta_{1}\left(U_{n}\right) \sum_{k=0}^{h} U_{n-k} \eta_{k}+\mu_{1}} \\
> & \frac{\lambda}{1+\mu_{1}+\beta_{1}\left(U_{n}\right) \sum_{k=0}^{h} U_{n-k} \eta_{k}} .
\end{aligned}
$$

Let $\beta_{1}^{M}\left(\epsilon_{0}\right)=\max _{U \in\left[0, \lambda / \mu_{1}+\epsilon_{0}\right]} \beta_{1}(U)$. Thus, we have

$$
\begin{aligned}
S_{n+1} & \geq \frac{\lambda}{1+\mu_{1}+\beta_{1}^{M} \sum_{k=0}^{h} U_{n-k} \eta_{k}} \\
& \geq \frac{\lambda}{1+\mu_{1}+\beta_{1}^{M}\left(\lambda / \mu_{1}+\epsilon_{0}\right) A} .
\end{aligned}
$$

Notice that $\epsilon_{0}$ can be arbitrarily small. Then, we have

$$
\begin{gathered}
\liminf _{n \rightarrow+\infty} S_{n+1} \geq m_{s}=\frac{\lambda}{1+\mu_{1}+\beta_{1}^{M}\left(A \lambda / \mu_{1}\right)}, \\
\beta_{1}^{M}=\max _{U \in\left[0, \lambda / \mu_{1}\right]} \beta_{1}(U) .
\end{gathered}
$$

Next, let us consider the positive sequences $S_{n}$ and $U_{n}$ of (2). According to these sequences, we define

$$
H_{n}=U_{n}+\frac{\mu_{2}+P+\xi_{1}}{A} \sum_{k=0}^{h}\left(\sum_{l=n-k}^{n} U_{l}\right) \eta_{k} \text {. }
$$

Then, for $n \geq 0$, we obtain

$$
\begin{aligned}
\Delta H & =H_{n+1}-H_{n} \\
& =U_{n+1}-U_{n}+\frac{\mu_{2}+P+\xi_{1}}{A} \sum_{k=0}^{h}\left(U_{n+1} \eta_{k}-U_{n-k} \eta_{k}\right) \\
& =\left(\beta_{1}\left(U_{n}\right) S_{n+1}-\frac{\mu_{2}+P+\xi_{1}}{A}\right) \sum_{k=0}^{h} U_{n-k} \eta_{k}+\beta_{3} U_{n+1} V_{n+1} .
\end{aligned}
$$

Since $R_{0}=\beta_{1}(0) A \lambda / \mu_{1}\left(\mu_{2}+P+\xi_{1}\right)>1$, there exist $0<\alpha<U_{\beta}$ and $\rho>0$ such that

$$
\begin{aligned}
& \frac{A \beta_{1}(0)}{\mu_{2}+P+\xi_{1}} \times \frac{\lambda}{\mu_{1}+\alpha \beta_{1}(\alpha) A} \\
& \quad \times\left\{1-\left(\frac{1}{1+\mu_{1}+\alpha \beta_{1}(\alpha) A}\right)^{\rho h}\right\}>1 ;
\end{aligned}
$$

note that

$$
S^{\Delta}=\frac{\lambda}{\mu_{1}+\alpha \beta_{1}(\alpha) A}\left\{1-\left(\frac{1}{1+\mu_{1}+\alpha \beta_{1}(\alpha) A}\right)^{\rho h}\right\} .
$$

We claim that it is impossible that $U_{n} \leq \alpha$ holds for all $n \geq n_{1} \geq[\rho h]$. The function $[x]$ gives the smallest integer not less than $x$. Suppose the contrary, for $n \geq n_{1}+h$. Consider

$$
\begin{aligned}
S_{n+1}= & \frac{\lambda+S_{n}+\xi_{1} U_{n+1}+\xi_{2} V_{n+1}}{1+\mu_{1}+\beta_{1}\left(U_{n}\right) \sum_{k=0}^{h} U_{n-k} \eta_{k}}>\frac{\lambda+S_{n}}{1+\mu_{1}+\alpha \beta_{1}(\alpha) A} \\
> & \frac{\lambda}{1+\mu_{1}+\alpha \beta_{1}(\alpha) A}\left\{1+\frac{1}{1+\mu_{1}+\alpha \beta_{1}(\alpha) A}\right. \\
& \left.+\left(\frac{1}{1+\mu_{1}+\alpha \beta_{1}(\alpha) A}\right)^{n-n_{1}-h-1}\right\} \\
& +\left(\frac{1}{1+\mu_{1}+\alpha \beta_{1}(\alpha) A}\right)^{n-n_{1}-h} S_{n_{1}+h+1} .
\end{aligned}
$$

From Lemma $1, S_{n}$ satisfies

$$
S_{n+1}>\frac{\lambda}{\mu_{1}+\alpha \beta_{1}(\alpha) A}\left\{1-\left(\frac{1}{1+\mu_{1}+\alpha \beta_{1}(\alpha) A}\right)^{n-n_{1}-h}\right\} \text {, }
$$

and we have that, for $n \geq n_{1}+h+[\rho h]$, we have

$$
\begin{aligned}
S_{n+1} & >\frac{\lambda}{\mu_{1}+\alpha \beta_{1}(\alpha) A}\left\{1-\left(\frac{1}{1+\mu_{1}+\alpha \beta_{1}(\alpha) A}\right)^{[\rho h]}\right\} \\
& \geq \frac{\lambda}{\mu_{1}+\alpha \beta_{1}(\alpha) A}\left\{1-\left(\frac{1}{1+\mu_{1}+\alpha \beta_{1}(\alpha) A}\right)^{\rho h}\right\}=S^{\Delta} .
\end{aligned}
$$

Hence, for $n \geq n_{1}+h+[\rho h]$, we have

$$
\begin{aligned}
\Delta H & \geq\left(\beta_{1}\left(U_{n}\right) S_{n+1}-\frac{\mu_{2}+P+\xi_{1}}{A}\right) \sum_{k=0}^{h} U_{n-k} \eta_{k} \\
& >\left(\beta_{1}(0) S^{\Delta}-\frac{\mu_{2}+P+\xi_{1}}{A}\right) \sum_{k=0}^{h} U_{n-k} \eta_{k} \\
& =\frac{\mu_{2}+P+\xi_{1}}{A}\left(\frac{A \beta_{1}(0) S^{\Delta}}{\mu_{2}+P+\xi_{1}}-1\right) \sum_{k=0}^{h} U_{n-k} \eta_{k} .
\end{aligned}
$$


Let $\epsilon=\min _{\theta}\left\{U_{n_{1}+[\rho h]+h+\theta} ; \theta=-h,-h+1, \ldots, 0\right\}$. Now, we will show that $U_{n} \geq \epsilon$ for all $n \geq n_{1}+[\rho h]+h$. In fact, there is an integer $\bar{n}>0$ such that

$$
\begin{gathered}
U_{n} \geq \epsilon, \quad n_{1}+[\rho h]+h \leq n \leq n_{1}+[\rho h]+h+\bar{n}, \\
U_{n+1}<\epsilon, \quad n=n_{1}+[\rho h]+h+\bar{n} .
\end{gathered}
$$

However, for $n=n_{1}+[\rho h]+h+\bar{n}$, we have

$$
\begin{aligned}
U_{n+1}-\epsilon= & \frac{\beta_{1}\left(U_{n}\right) S_{n+1} \sum_{k=0}^{h} U_{n-k} \eta_{k}+U_{n}}{1+\mu_{2}+P+\xi_{1}-\beta_{3} V_{n+1}}-\epsilon \\
\geq & \frac{\beta_{1}\left(U_{n}\right) S_{n+1} \sum_{k=0}^{h} U_{n-k} \eta_{k}+U_{n}}{1+\mu_{2}+P+\xi_{1}} \\
& -\frac{1+\mu_{2}+P+\xi_{1}}{1+\mu_{2}+P+\xi_{1}} \epsilon \\
\geq & \frac{\beta_{1}(0) S^{\Delta} A-\left(\mu_{2}+P+\xi_{1}\right)}{1+\mu_{1}+P+\xi_{1}} \epsilon \\
= & \frac{\mu_{2}+P+\xi_{1}}{1+\mu_{2}+p+\xi_{1}}\left\{\frac{\beta_{1}(0) S^{\Delta} A}{\mu_{2}+P+\xi_{1}}-1\right\} \epsilon>0 .
\end{aligned}
$$

Which is a contradiction. Thus, $U_{n} \geq \epsilon$ for $n \geq n_{1}+[\rho h]+h$. Therefore, for $n \geq n_{1}+[\rho h]+h$,

$$
\Delta H>\left(\mu_{2}+P+\xi_{1}\right)\left\{\frac{A \beta_{1}(0) S^{\Delta}}{\mu_{2}+P+\xi_{1}}-1\right\} \epsilon>0,
$$

which implies that $H_{n} \rightarrow+\infty$ as $n \rightarrow+\infty$. But, from Lemma 2 and (46), there exists a sufficiently large integer $n_{1}^{\prime}>0$ such that, for $n>n_{1}^{\prime}$,

$$
\begin{aligned}
H_{n} & \leq \frac{\lambda}{\mu_{1}}+\frac{\mu_{2}+P+\xi_{1}}{A} \sum_{k=0}^{h}\left(\sum_{l=n-k}^{n} \frac{\lambda}{\mu_{1}}\right) \eta_{k} \\
& \leq \frac{\lambda}{\mu_{1}}\left\{1+\left(\mu_{2}+P+\xi_{1}\right)(h+1)\right\},
\end{aligned}
$$

which is a contradiction. Hence, the claim is proved.

In the rest, we only need to consider the following two cases:

(i) $U_{n} \geq \alpha$ for all large $n$.

(ii) $U_{n}$ oscillates about $\alpha$ for all large $n$.

We show that $U_{n} \geq m_{u}$ for all large $n$, where $0<m_{u} \leq$ $\alpha$, is a constant which will be given later. Clearly, we only need to consider case (ii). Let positive integers $n_{1}$ and $n_{2}$ be sufficiently large that $U_{n_{1}} \geq \alpha, U_{n_{2}} \geq \alpha$, and $U_{n}<\alpha$, for $n_{1}<n<n_{2}$.

$$
\begin{aligned}
& \text { If } n_{2}-n_{1}<h+[\rho h] \text {, since } \\
& U_{n}=\frac{\beta_{1}\left(U_{n-1}\right) S_{n} \sum_{k=0}^{h} U_{n-k-1} \eta_{k}+U_{n-1}}{1+\mu_{2}+P+\xi_{1}-\beta_{3} V_{n}} \\
& \geq \frac{U_{n-1}}{1+\mu_{2}+P+\xi_{1}},
\end{aligned}
$$

we have

$$
\begin{aligned}
U_{n} & \geq\left(\frac{1}{1+\mu_{2}+P+\xi_{1}}\right)^{n-n_{1}} U_{n_{1}} \\
& >\left(\frac{1}{1+\mu_{2}+P+\xi_{1}}\right)^{n_{2}-n_{1}} U_{n_{1}} \\
& >m_{u}=\left(\frac{1}{1+\mu_{2}+P+\xi_{1}}\right)^{h+[\rho h]} \alpha .
\end{aligned}
$$

Hence, $U_{n}>m_{u}$ for $n \in\left[n_{1}, n_{2}\right]$.

If $n_{2}-n_{1}>h+[\rho h]$, we can easily obtain that $U_{n}>m_{u}$ for $n \in\left[n_{1}, n_{1}+h+[\rho h]\right]$. Assume that there exists an integer $\widehat{n}>0$ such that

$$
\begin{gathered}
U_{n} \geq m_{u}, \quad n_{1}+h+[\rho h] \leq n \leq n_{1}+h+[\rho h]+\widehat{n}, \\
U_{n+1}<m_{u}, \quad n=n_{1}+h+[\rho h]+\widehat{n} .
\end{gathered}
$$

However, for $n=n_{1}+h+[\rho h]+\widehat{n}$,

$$
U_{n+1}-m_{u} \geq \frac{\mu_{2}+P+\xi_{1}}{1+\mu_{2}+p+\xi_{1}}\left\{\frac{\beta_{1}(0) S^{\Delta} A}{\mu_{2}+P+\xi_{1}}-1\right\} m_{u}>0 .
$$

This is a contradiction to the proposition that $U_{n+1}<$ $m_{u}$. Therefore, $U_{n} \geq m_{u}$ for $n \in\left[n_{1}, n_{2}\right]$. Since these positive integers $n_{1}$ and $n_{2}$ are chosen in an arbitrary way, we conclude that $U_{n} \geq m_{u}$ for all large $n$ in case (ii). Hence, $\liminf _{n \rightarrow \infty} U_{n} \geq m_{u}$.

Note that, from that third equation of system (2), we have

$$
\liminf _{n \rightarrow \infty} V_{n} \geq m_{v}=\frac{P}{\mu_{3}+\xi_{2}+\lambda \beta_{3} / \mu_{1}} m_{u}=\frac{P}{\mu_{3}+\xi_{2}+\beta_{3} S^{0}} m_{u}
$$

From Lemma 2 and the discussion above, we have

$$
\begin{aligned}
& m_{s} \leq \liminf _{n \rightarrow \infty} S_{n} \leq \limsup _{n \rightarrow \infty} S_{n} \leq S^{0}, \\
& m_{u} \leq \liminf _{n \rightarrow \infty} U_{n} \leq \limsup _{n \rightarrow \infty} U_{n} \leq S^{0}, \\
& m_{v} \leq \liminf _{n \rightarrow \infty} V_{n} \leq \limsup _{n \rightarrow \infty} V_{n} \leq S^{0} .
\end{aligned}
$$

The proof is completed. 

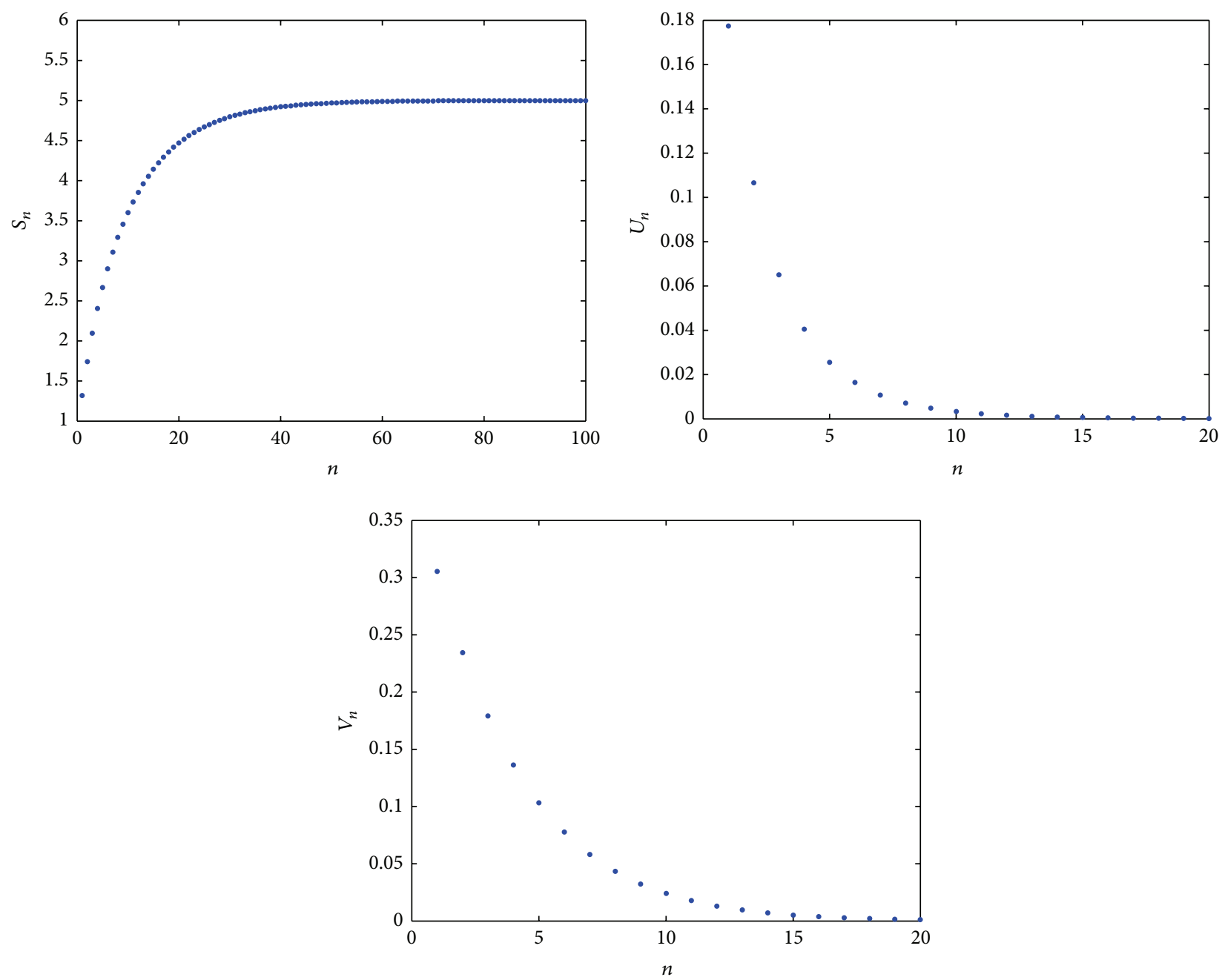

FIGURE 1

\section{Numerical Example}

In order to confirm the validity of our results, we consider the following heroin epidemic model with a discrete time delay:

$$
\begin{aligned}
S_{n+1}-S_{n}= & \lambda-\beta_{1} S_{n+1} U_{n-h}-\mu_{1} S_{n+1}+\xi_{1} U_{n+1}+\xi_{2} V_{n+1}, \\
U_{n+1}-U_{n}= & \beta_{1} S_{n+1} U_{n-h}+\beta_{3} U_{n+1} V_{n+1} \\
& -\left(\mu_{2}+P+\xi_{1}\right) U_{n+1} \\
V_{n+1}-V_{n}= & P U_{n+1}-\beta_{3} U_{n+1} V_{n+1}-\left(\mu_{3}+\xi_{2}\right) V_{n+1} .
\end{aligned}
$$

Now, we present a numerical example. For the sake of simplicity, we choose the parameters as $\beta_{1}=0.9, \beta_{3}=0.8$, $\lambda=2, \mu_{1}=0.1, \mu_{2}=0.2, \mu_{3}=0.1, P=0.4, \xi_{1}=0.1$, and $\xi_{2}=0.2$; we get $R_{0}=25.7143<1$. Figure 1 shows that the disease free equilibrium $E^{0}$ of the system (64) is globally asymptotically stable when $R_{0}<1$. Figure 2 shows that the system (64) is permanent when $R_{0}>1$.

\section{Conclusions}

In this paper, we have modified the Samanta heroin epidemic model into an autonomous heroin epidemic model with distributed time delay. Further, we established a discretized heroin epidemic model with time delay, sufficient conditions have been obtained to ensure the global asymptotic stability of heroin-using free equilibrium when $R_{0} \leq 1$ and $\beta_{1}(U)$ is replaced by a positive constant. We also carried out some discussion about the heroin-using equilibrium, but our results are only restricted to the existence of this equilibrium for $\beta_{1}(U)=\beta_{1}>0$, a special case of system (2). The stability of heroin-using equilibrium is yet to be studied. As a main result of this paper, we obtained the permanence of the system (2). From the expression of $R_{0}=\beta_{1}(0) \lambda A / \mu_{1}\left(\mu_{2}+P+\xi_{1}\right)$, we see that a decrease in $\beta_{1}$ (transmission coefficient from susceptible population) will cause a decrease of the same proportion in $R_{0}$. If the rate of migration or recruitment is restricted into susceptible community, the spread of the disease can also be kept under control by reducing $\lambda$ and thereby decreasing $R_{0}$. The spread of the heroin users can also be controlled by educators, epidemiologists, and treatment 

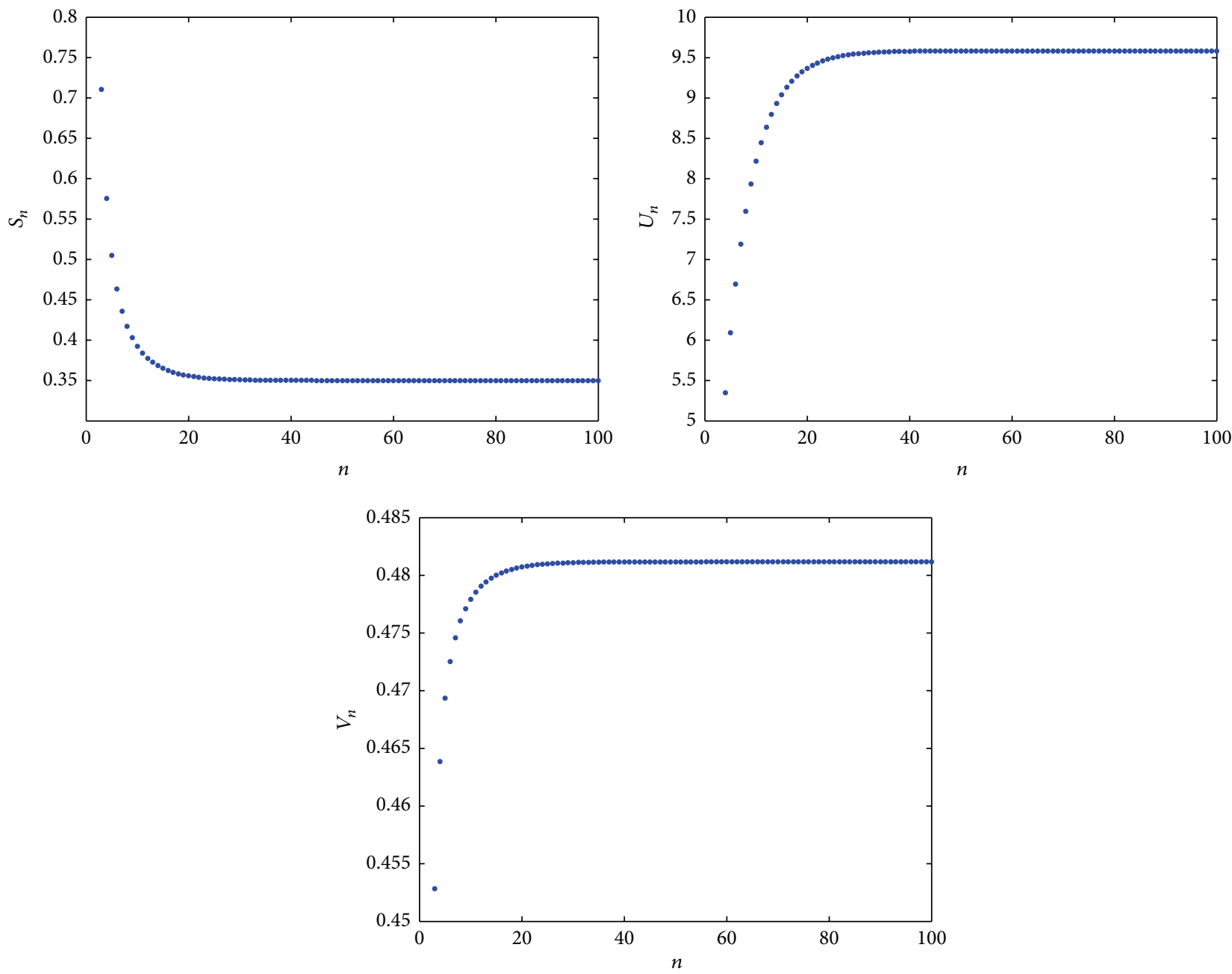

FIGURE 2

providers to increase the values of $\xi$ (removal rate of heroin users not in treatment who stop using heroin but are susceptible) and $P$ (proportion of heroin users who enter treatment) and thereby to decrease $R_{0}$. This analysis tells us that prevention is better than cure; efforts to increase prevention are more effective in controlling the spread of habitual drug use than efforts to increase the numbers of individuals accessing treatment.

\section{Conflict of Interests}

The authors declare that there is no conflict of interests regarding the publication of this paper.

\section{Acknowledgment}

This work was supported by the National Natural Science Foundation of China (Grants nos. 11261056, 11261058, and 11271312).

\section{References}

[1] European Monitoring Centre for Dru gs and Drug Addiction (CMCDDA), “Annual Report, 2005," http://www.emcdda .europa.eu/publications/annual-report/2005.

[2] C. Comiskey, "National prevalence of problematic opiate use in Ireland," Tech. Rep., EM-CDDA, 1999.

[3] A. Kelly, M. Carvalho, and C. Teljeur, Prevalence of Opiate Use in Ireland 2002-2001. A 3-Source Capture Recapture Study. A Report to the National Advisory Committee on Drugs, Subcommittee o $n$ Prevalence, Small Area Health Research Unit, Department of Public, 2003.

[4] K. A. Sporer, "Acute heroin overdose," Annals of Internal Medicine, vol. 130, no. 7, pp. 584-590, 1999.

[5] E. White and C. Comiskey, "Heroin epidemics, treatment and ODE modelling," Mathematical Biosciences, vol. 208, no. 1, pp. 312-324, 2007.

[6] W. Wang, "Global behavior of an SEIRS epidemic model with time delays," Applied Mathematics Letters, vol. 15, no. 4, pp. 423428, 2002. 
[7] P. van den Driessche and J. Watmough, "Reproduction numbers and sub-threshold endemic equilibria for compartmental models of disease transmission," Mathematical Biosciences, vol. 180, pp. 29-48, 2002.

[8] T. Zhang and Z. Teng, "Global behavior and permanence of SIRS epidemic model with time delay," Nonlinear Analysis: Real World Applications, vol. 9, no. 4, pp. 1409-1424, 2008.

[9] D. R. Mackintosh and G. T. Stewart, "A mathematical model of a heroin epidemic: Implications for control policies," Journal of Epidemiology and Community Health, vol. 33, no. 4, pp. 299304, 1979.

[10] H. Su and X. Ding, "Dynamics of a nonstandard finitedifference scheme for Mackey-Glass system," Journal of Mathematical Analysis and Applications, vol. 344, no. 2, pp. 932-941, 2008.

[11] M. Sekiguchi and E. Ishiwata, "Global dynamics of a discretized SIRS epidemic model with time delay," Journal of Mathematical Analysis and Applications, vol. 371, no. 1, pp. 195-202, 2010.

[12] G. Mulone and B. Straughan, "A note on heroin epidemics," Mathematical Biosciences, vol. 218, no. 2, pp. 138-141, 2009.

[13] J. Liu and T. Zhang, "Global behaviour of a heroin epidemic model with distributed delays," Applied Mathematics Letters, vol. 24, no. 10, pp. 1685-1692, 2011.

[14] G. Huang and A. Liu, "A note on global stability for a heroin epidemic model with distributed delay," Applied Mathematics Letters, vol. 26, no. 7, pp. 687-691, 2013.

[15] G. P. Samanta, "Dynamic behaviour for a nonautonomous heroin epidemic model with time delay," Journal of Applied Mathematics and Computing, vol. 35, no. 1-2, pp. 161-178, 2011.

[16] X. Wang, J. Yang, and X. Li, "Dynamics of a Heroin epidemic model with very population," Applied Mathematics, vol. 2, no. 6, pp. 732-738, 2011.

[17] R. E. Mickens, "Discretizations of nonlinear differential equations using explicit nonstandard methods," Journal of Computational and Applied Mathematics, vol. 110, no. 1, pp. 181-185, 1999. 


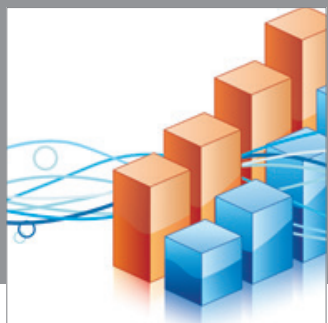

Advances in

Operations Research

mansans

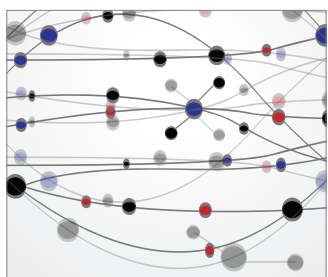

The Scientific World Journal
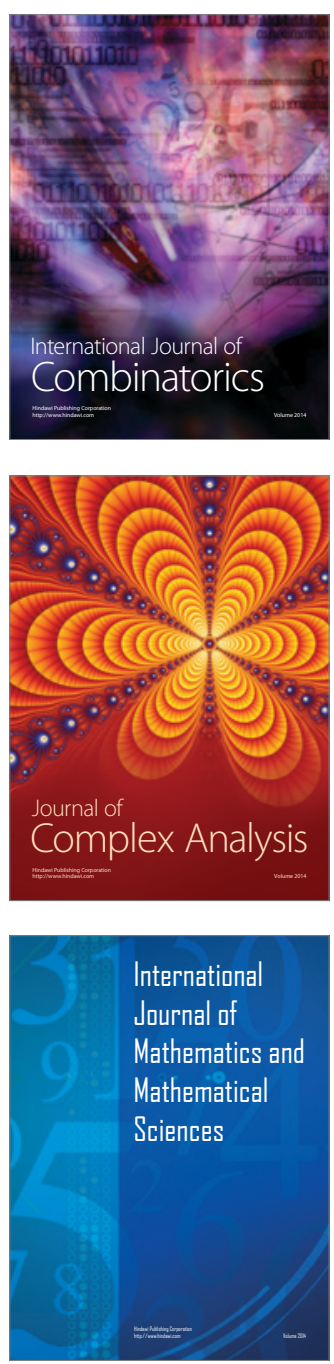
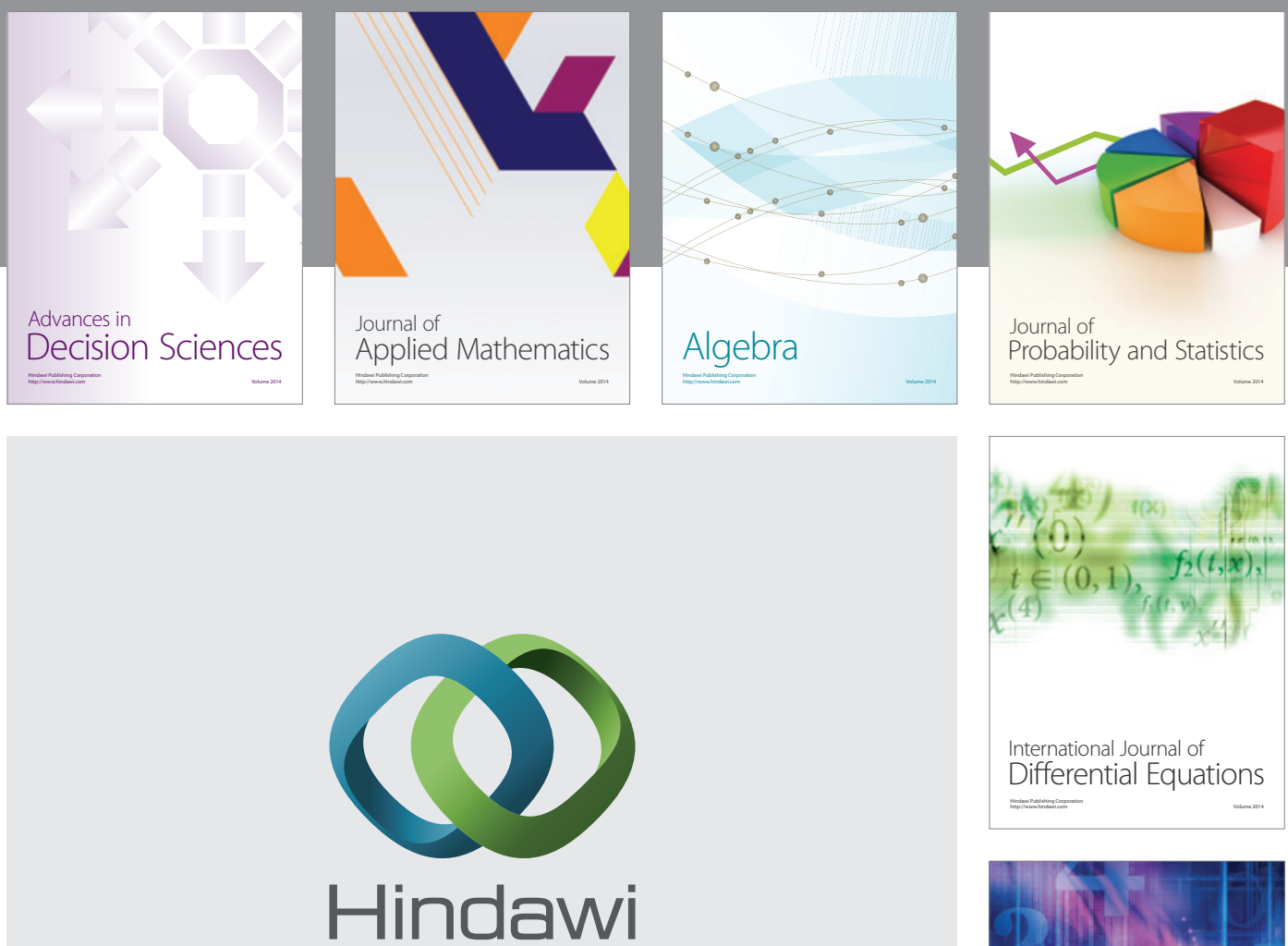

Submit your manuscripts at http://www.hindawi.com
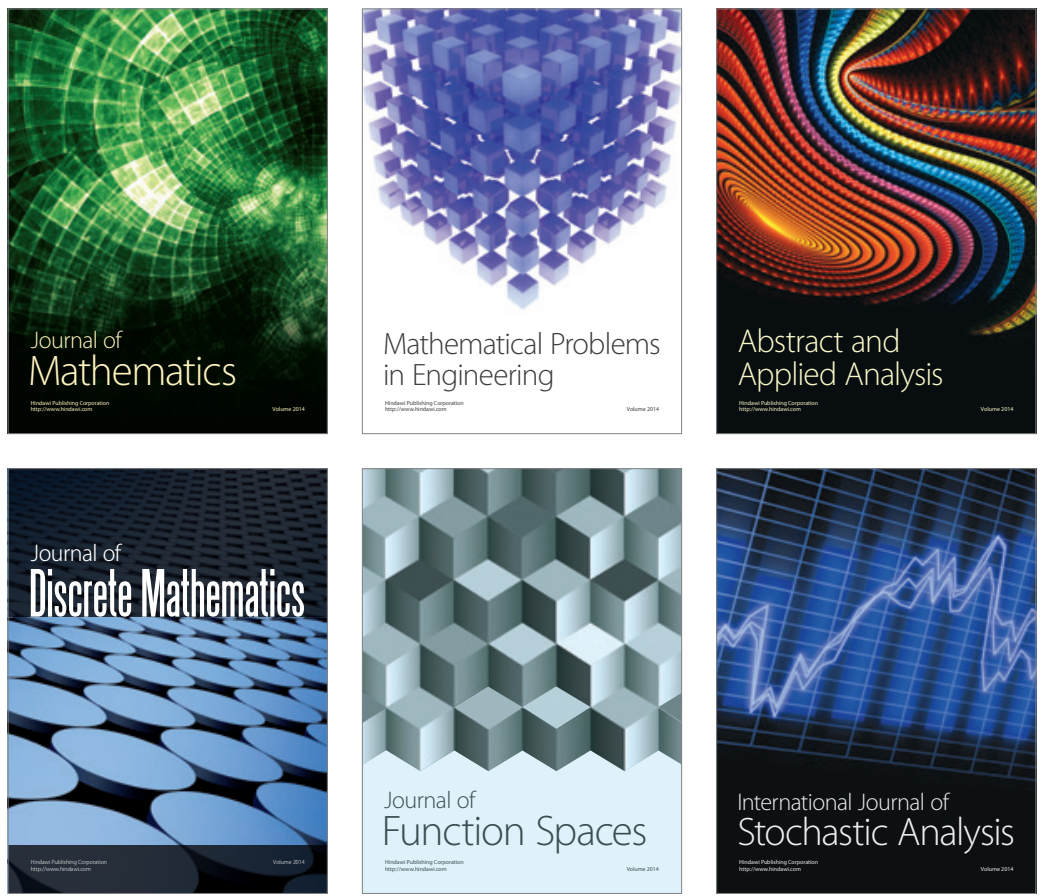

Journal of

Function Spaces

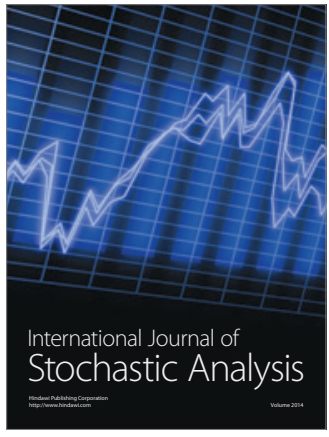

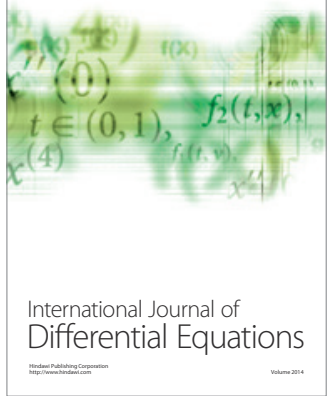
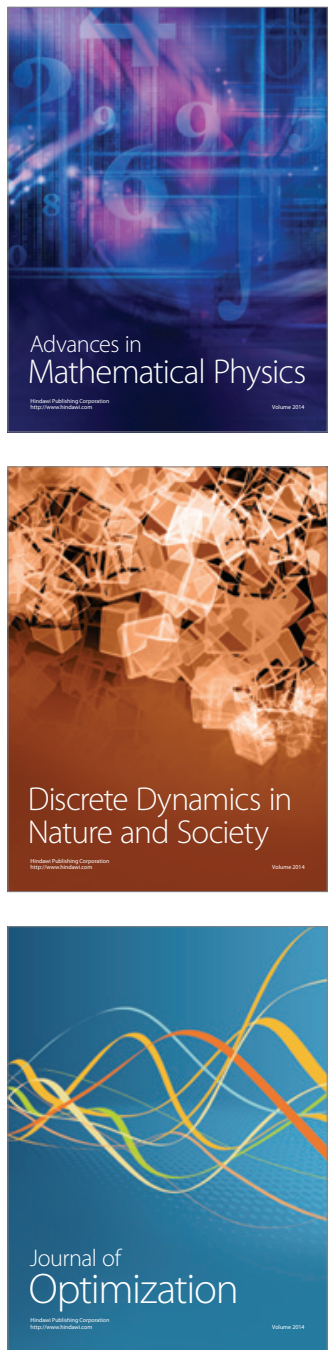